therapy as described by Garner." Lastly, perhaps more attractive student electives could be devised at psychiatric establishments both at home and abroad. Interestingly, in Newcastle, Mental Health Foundation grants have been made available to a number of students and, following electives in psychiatry, all have pursued a career in the speciality.

Many developed their interest in psychiatry at a later stage and recent schemes such as the pre-registration psychiatry posts in Sheffield, ${ }^{6}$ have important implications particularly as Parkhouse \& Dartun ${ }^{7}$ have suggested that after extended exposure to the speciality the drop-out rate is low.

In the long term, psychiatrists obviously have a key role in enhancing recruitment. If the attitudinal shift shown by many of the students is maintained in the future it may be easier for some potential trainees to enter the speciality. However, this will not happen unless psychiatrists commit themselves to active and enthusiastic recruitment. Ultimately, this commitment must be matched by an equal determination to extend our understanding of the disorders we treat and to receive adequate support in these endeavours. It is perhaps still pertinent to consider the comment of Ellis, ${ }^{8}$ made over 20 years ago- The fact that
$40 \%$ of British hospital beds are occupied by psychiatric patients will never lead students to be interested in psychiatry as will some therapeutic advance which empties these beds'.

REFERENCES

'Brook, P. (1983) Who's for psychiatry? United Kingdom medical schools and career choice of psychiatry, 1961-75. British Journal of Psychiatry, 142, 361-365.

${ }^{2}$ Kartun, D. (1983) Psychiatry in anguish. World Medicine, Jan. $22,46$.

${ }^{3}$ MorRes, P. (1982) The poor image of psychiatrists. World Medicine, Feb. 6, 101-103.

4CREED, F. (1979) Who Puts Medical Students Off Psychiatry? Welwyn Garden City: Smith Kline \& French Publications.

${ }^{5}$ Garner, P. (1981) Psychotherapy: Experience as a medical student. British Medical Journal, 282, 797.

${ }^{6}$ Seager, C. P. (1985) Pre-registration house officer posts in psychiatry. Bulletin of the Royal College of Psychiatrists, 9, $141-142$.

'PARXrouse, J. M. \& Dartun, R. A. (1979) Training in the hospital specialities in Britain in 1975. British Medical Journal, 1, 670.

${ }^{8}$ ELLu, J. (1963) The teaching of psychiatry. British Medical Journal. 2, 585-588.

\title{
Second Annual Meeting of the Society for Psychotherapy Research
}

Graeme McGrath, Lecturer in Psychiatry, University Hospital of South Manchester and

StEVE ReILly, Senior Registrar in Psychotherapy, Regional Psychotherapy Unit, Gaskell House, Swinton Grove, Manchester

The United Kingdom branch of the Society for Psychotherapy Research SPR(UK) held its second annual meeting at Ravenscar in North Yorkshire in April 1985. The setting, as in 1984, was a hotel high on a cliff overlooking Robin Hood's Bay, where conference members with a few minutes to spare could walk in the clifftop gardens or attempt the arduous climb down to the beach. No one took advantage of the golf course this year, nor apparently was anyone hardy enough to swim in the open-air pool!

The meeting was divided into six plenary sessions each with a common theme and three smaller workshops. Presentations were of a uniformly high standard and ranged from reports of small-scale research projects carried out by individuals in the course of everyday clinical work to large-scale projects with elaborate experimental design and teams of investigators. The message was clear: valuable psychotherapy research can be and is being done in busy NHS settings and well-endowed academic institutions alike, and moreover is being carried out by psychotherapists from a variety of professional backgrounds including psychiatry, clinical psychology and social work; a combination guaranteed to provide a fertile substrate for the generation and testing of new ideas.
It is not possible to discuss psychotherapy research for long without considering the related issues of process and outcome, and this was the theme of the opening session, devoted to work derived from the Sheffield Psychotherapy Research Project. David Shapiro discussed this comparative study of prescriptive versus exploratory psychotherapy using a sophisticated crossover design in which therapist variables were controlled by having the same therapist deliver both modes of therapy to the same patient. This work shows that both exploratory and prescriptive modes of therapy are effective and also that the outcome differs depending on the order in which treatments are given. This is clearly an important finding when considering studies which seem to show little difference in effect between different treatments. Shapiro was the first of several presenters to point out that when considering service delivery and priorities in research the issue of cost effectiveness must be taken into account.

Jenny Firth described some of the techniques used in the research and gave clinical examples. Bill Styles, using the same pool of patients, described his work on the immediate impact of individual sessions as judged by patients and therapists. Session impact is a separate concept from both 
process and outcome and its importance is as a possible intermediary between the two. Interestingly there was a very low correlation between patients' and therapists' judgements about the impact of sessions, but the therapists' evaluation was a better predictor of overall outcome. Sue Llewellyn and Robert Elliot extended the 'what makes psychotherapy effective?' debate by looking at helpful events in sessions both quantitatively using questionnaires and qualitatively using the technique of comprehensive process analysis of audio tapes. Across all sessions problem solution and awareness events were found to be most helpful regardless of the treatment mode, but problem solution was more helpful in the prescriptive mode and increased awareness helpful in the exploratory mode, suggesting a real difference between therapies in their mode of action. Detailed analysis of 'insight' events perceived as helpful suggests that there are features (therapist interpretation of core conflicts delivered in a persistent but interactive style) common to both therapies. Overall the differences between prescriptive and exploratory therapies were consistent with their theoretical basis, and similarities were closely related to therapist factors, again suggesting very real differences between different forms of psychotherapy.

The second session concentrated on the boundary between social psychology and psychotherapy research. Mikacl Leiman described the difficulties of conducting research into a service provided to a small rural community literally in the backwoods of Finland, where traditional social structures are undergoing rapid change and diagnostic systems developed in the rest of Europe may have little relevance. He discussed a multiaxial approach to diagnosis that attempts to encompass psychological, social, and theoretical approaches to mental disturbance. Sue Llewellyn and Guy Fielding offered a perspective on the social psychology of groups, suggesting that clinicians in all disciplines tend to concentrate on the individualistic ways of analysing process, even in therapeutic groups, and they propose that a social psychology approach could permit a valuable new perception of group processes in clinical settings. David Pilgrim described a wide range of sociological and philosophical perspectives on the use of accounts in human science research, and described his own use of detailed accounts from 12 dynamic psychotherapists in the collation of a list of important themes in their perception of their own practice. Krysia Yardley pursued a topic that was prominent during last year's meeting, the so-called 'new paradigm research'. She emphasised the need to take a personal approach to the assumptions of every individual psychotherapist and researcher, describing possible ways of exploring the values of therapists and researchers and making these explicit. She hypothesised that change in psychotherapy might involve the patient's acceptance of the therapist's moral values.

In the third session Professor Israel Kolvin described the 12-year Newcastle study which examines the effects of different psychotherapies in childhood disorders. Both behaviour modification and group therapy proved signifi- cantly better than either the control, nurturing or parent counselling groups, despite being of shorter duration than either of the ineffective interventions. These improvements were sustained for at least 18 months after treatment ended, and Professor Kolvin concluded that 'a new set of processes were set in train' by psychotherapy. The problem of chronic aggressiveness in children and the cycles of abuse in families formed the basis of Rory Nicol's presentation of a joint project between the University and the NSPCC Special Unit in Newcastle. Using interactive behaviour ratings in families they have identified 14 'coercive' categories of behaviour and developed a focused casework procedure aimed at changing these rigid patterns. Coercive behaviours were reduced in all family members during the study but seemed to increase in mothers after intervention ended. A further study including measures of stress on mothers is currently under way.

Ivan Eisler, from the Institute of Psychiatry, reviewed a team project comparing family and individual psychotherapy in patients with anorexia nervosa, and presented one year follow-up data. Overall there was little difference between the therapeutic modes but younger patients tended to do better with family therapy and older patients tended to show more benefit from longer term individual therapy. The session ended with Bernie Rosen and Angela Summerfield showing a video tape illustrating the methodology of simultaneous multi-camera recording to produce frame by frame analyses of non-verbal interactions in families, which can be analysed on relationship grids to define the constellation of family communication patterns in more detail than in single recording.

The majority of minor psychiatric disorders present to general practitioners and so the relationship between psychotherapy and general practice is of considerable importance. This relationship was explored in the next plenary session which began with John Cope who emphasised that as the majority of minor psychiatric disorders are dealt with in general practice settings, it is in comparison with GP treatment that psychotherapy must prove itself to be a cost-effective treatment for such disorders. It is therefore essential that we define the therapeutic components of GP treatment, elucidation of which could also help GPs become more effective. Cope is currently engaged in a study of individual patient consultations with GPs for psychological problems, tape recording interviews and studying content, verbal response modes and process. Justin Livingstone, himself a general practitioner, described the arrangements necessary for the effective attachment of a psychotherapist to a general practice and Jane Mounty discussed the evaluation of the attachment of a therapist to Dr Livingstone's practice. Patients were assessed for suitability for brief psychotherapy and were then randomly allocated to treatment by a dynamically orientated psychotherapist or by their own GP. At present only the early follow-up assessments are available and both groups had made significant improvements. The patients treated with psychotherapy reported more satis- 
faction with treatment and showed more improvement in their social adjustment. They also made less visits to their GP during the follow-up period, a finding consistent with other studies and which has important cost implications. Jean Arundale has looked at a psychotherapy service in the community, in which clients are seen in their own homes. Although the number of clients was small her data indicated that treatment produced improvement in a range of outcome measures, and that good results were not necessarily related to intense, focused treatment.

Beginning a mixed bag of unrelated presentations later in the day, Professor Rachel Rosser presented further data from the Cassel Hospital follow-up study which included economic evaluation of outcome. Both subjective health status and economic productivity were highly correlated with clinical outcome. Conservative economic evaluation shows that patients with a good outcome showed a net 'profit' at five-year outcome, whereas poor outcome patients showed a net loss. This indicates the importance both of formal economic evaluation, which can indicate clearly the best treatment strategy to adopt, and the importance of identifying in advance those patients who are likely to benefit from treatment. Failure to identify such characteristics led Rosser to review the casenotes of all patients in the study. The factor that best predicted successful outcome was clients having spent eight weeks on the waiting list before admission. This was predictive of employment status, subjective health status, symptomatic improvement and reduced medical utilisation and was not simply due to a period on the waiting list selecting more motivated clients. The waiting list theme was continued by Lee Barnes, who has been attempting to assess the experience of being on a waiting list (for a clinical psychology service) that could be as long as two years! As might be expected, those clients who waited 'passively', making no effort to cope with their problems, were less likely to improve while on the waiting list and also had lower expectations of treatment. There is considerable evidence that untreated minor psychiatric disorders tend to become chronic and can constitute a considerable drain on NHS resources. Studying these issues from an economic perspective would demonstrate whether it is cost effective to increase resources to the psychology and psychotherapy services to reduce excessively long waiting lists.

In the next presentation Monica Davies, Robert Elliot and John Davis represented several other members of a collaborative multi-centre project aimed at developing a taxonomy of 'therapist difficulties' which they hope will be relevant to development of theory, therapist training and the improvement of clinical practice.

Sometimes therapists are accused of mystifying their patients and Polly Crisp has developed a coding manual for measuring 'mystification' from tape recordings of therapy sessions. She found that some therapists used cryptic communications unrelated to the patient's utterances. Such mystifying communication was consistently used by therapists with several patients and was significantly related to worse outcome in schizophrenic patients receiving psychotherapy as part of their treatment. Duncan Cramer ended the session with an investigation of Carl Roger's 'therapeutic factors' of genuineness, warmth, empathy and positive regard. Self-esteem and factors in the quality of relationships were studied in college students and their friends using the Barret-Lennard Relationship Inventory. Partial correlation indicated that positive regard was the only factor significantly associated with self-esteem in subjects and cross-lagged panel correlation techniques further suggested that self-esteem was secondary to an individual's subjective perception of others' regard.

The final session began with the presentation, by Sidney Bloch and Eric Crouch, of a new operational classification of therapeutic factors in group psychotherapy evolved from an exhaustive literature review. They urged that the theory of specific therapeutic factors in group psychotherapy needs to be underpinned by more effective research, and suggested a number of areas for such research. They argue for a more accurate specification of research variables and the need for research to be cumulative, so that each stage develops from previous work in a logical manner. To conclude, Mary Burton gave a fascinating description of the massive regression exhibited by students during a weekend group dynamic workshop, using both clinical examples and her own technique for measuring the degree of regression from tapes of sessions.

There was also time allocated to small workshops, where conference members could choose one of three alternatives. Frank Margison convened a workshop on aspects of teaching and research in psychotherapy, Robert Elliot led discussion on the analysis of significant therapy events using his own tape-recorded material, and Bill Styles presented an introduction to Verbal Response Mode coding. In summing up, this was an intensive and rewarding conference packed with good presentations of high quality work.

Further information about $S P R(U K)$ can be obtained from Professor J. Watson, Department of Psychiatry Guy's Hospital Medical School, St Thomas St, London SEI 9RT.

\section{Research Register}

The Team for the Assessment of Psychiatric Services (TAPS) was set up in 1985 by the North East Thames Regional Health Authority in order to evaluate their policy to close Claybury Hospital and partially close Friern Hospital. As part of their work, the team has set up a register of research, to include current studies of the transfer of care from psychiatric hospitals to districts. Although focused on the North East Thames Region, their remit includes related research in other geographical areas. They would welcome information and enquiries about the research register, which will be circulated to interested parties at regular intervals. Please contact Dr Julian Leff, Honorary Director TAPS, Research Unit, Friern Hospital, Friern Barnet Road, London N11 3BP. 